\title{
Presenting Research in a Scientific Article
}

\author{
Jo. M. BARNES
}

\begin{abstract}
Criteria are set out that referees can use to judge manuscripts offered for publication. One of the criteria is clarity of the writing and soundness of the organisation of the article. This paper deals mainly with this requirement of good writing and logical organisation and sets out the broad structure of a scientific article under the various headings usually encountered in research publications. Hints are given to help the author to organise the mass of information into a logical and readable form, while keeping the requirements of electronic searching of literature in databases in mind.
\end{abstract}

\section{INTRODUCTION}

Concern has been voiced in many quarters about the increasing number of poorly written scientific articles appearing in biomedical journals.

There are many reasons for this disquieting situation. The frequently invoked "publish or perish" syndrome certainly sometimes results in premature publication of ill-conceived research in poorly written articles, but cannot be blamed for all of it. Part of the problem also rests with scientists, who are not well acquainted with all the implications of research methodology, statistical analysis and presentation of research in scientific articles. This article is aimed at providing information about the organisation of original research or clinical material in a scientific article.

\section{CRITERIA FOR JUDGING MANUSCRIPTS}

Before turning to the structure of the scientific article and how to organise the information in it clearly and logically, it may be worthwhile looking at the criteria referees are recommended to use when judging the merits of manuscripts to be published.

In South Africa, like any country with a relatively small research community, the authors and the pool of possible referees of scientific papers consist to a large extent of the same people. From this it follows that, should the writing expertise in any particular research field be lacking, the refereeing process will be suboptimal as well, since the same inexperienced authors are called upon to judge the papers of their fellow scientists. This is another reason why standards of published research are dropping.

Jo M. Barnes, Institute for Medical Literature, Medical Research Council
Formal criteria for judging manuscripts submitted for publication can guide experienced and inexperienced referees alike. These criteria may help inexperienced referees by making them aware of various aspects to look out for in manuscripts, and can serve as a checklist for experienced referees to avoid omissions. Especially referees who judge manuscripts for a variety of journals with different requirements may welcome a set of suggested criteria from the editor. Ideally these criteria should include: 1

- the importance of the research question or subjectfield studied,

- originality of the work,

- appropriateness of the approach or experimental design,

- adequacy of the experimental techniques,

- soundness of the conclusions and interpretation,

- relevance of the discussion,

- clarity of the writing and soundness of the organisation of the paper.

This article will be concerned mainly with the lastmentioned criterion. Organising the content of a scientific article can present problems even to experienced authors. No two scientific articles ever pose identical problems to the author and it is not possible to lay down rigid and unalterable rules by which to present the information. Nevertheless, over the past three centuries of published science a broad structure has developed for disseminating biomedical research findings to fellow scientists that represents the most logical and streamlined order of information for the reader. The 1 main consideration in writing up research findings is the logical understanding of the reader and not ease of writing for the author.

\section{STYLE OF A SCIENTIFIC ARTICLE}

A scientific article is not a literary work and does not follow the same style. Clarity is essential. The content of a scientific article is complex and the terminology sophisticated. Therefore, keep sentences as short as possible without resorting to a telegraphic style. Avoid the passive voice and remove all unnecessary jargon or repetition of the same concept (tautology). It wastes space and irritates the reader. Long, meandering sentences obscuring the meaning or complicating the reading task are particularly detrimental to the objective of a scientific'article, namely the carrying over of carefully weighed information. 


\section{SEQUENCE OF INFORMATION}

The presentation of scientific research in an article is largely a question of organisation. Each article should, in the right sequence, contain sections usually called Introduction, Materials and Methods, Results, Discussion (Conclusions), and References. Any other sequence will present problems to the reader. In essence all these parts or sections are always present in a good article, but not all articles lend themselves to the specific wording of the above-mentioned headings. The titles of these sections can be adapted to suit the individual study. These sections should never be omitted though - it is inconceivable that research can be reported without a description of, for instance, the methods and the results.

In the following discussion the various headings used in scientific articles will be dealt with in the order that they appear in the final manuscript. This is not the order in which these individual pieces are written by the author, though. For instance, the final title and the abstract appear at the top of the article, but it should be drawn up last of all - after the manuscript is written in its entirety.

\section{CONSTRUCTING A TITLE}

The title of a scientific article is most important. This is the only part thousands of people will read with certainty and can determine whether the whole article will be read.

Titles should be as short as possible, but yet so specific as to convey the contents of the article accurately. There is a danger, though, attached to titles that are too short. These titles are usually constructed of words that are too vague or too broad in meaning. "The action of antibiotics on bacteria" is too vague. It is not the title of a paper, but more likely to be the title of a book or series of books. Each broad, vague term can be replaced by, for example, specific terms like "The action of some antibacterial compounds on Staphylococcus aureus". That can in some cases still be too vague and "action" can be described further (for example "inhibition of growth"). The antibacterial compounds can be named or the broad generic groups of drugs stated, if too numerous to mention all names. By expanding the title in this way - starting with a short but broad sentence and expanding each term - the syntax is not disturbed and it is easy for an inexperienced writer to place all the terms in logical order.

The title of an article is its label. It is used to retrieve the article by means of computerised searches or to categorise the article according to its contents. In the expansion of the title try to use the key words under which you would search for such an article.

Avoid "clever" titles or titles trying to catch the reader's attention by journalistic techniques. Rather be prosaic, but specific. Few readers come across articles by browsing in the library these days. Articles are brought to the attention of potential readers through computerised literature searches or abstracting services. By choosing a tantalising but vague title like "Physio- therapy - the road ahead" the article may be filed in the data base never to be retrieved again. If extra key words describing the contents more accurately are not allocated to the article, no search will turn up an article under such a title.

\section{THE LIST OF AUTHORS}

This is an easy writing task, but a difficult decision. There is no objective "right" sequence of listing the authors. Some laboratories and institutions place the authors alphabetically. This has obvious drawbacks. A safe rule is to look at the contributions of the various authors to the research and to list them in the order of importance of their contributions. The influential Council for Biology Editors states in its latest Style Manual:2 "Do not list as an author any person who has not participated conceptually and materially in planning, executing, or analyzing the research. An early decision about authorship may prevent later misunderstanding and embarrassment. The practice of including in the byline (author designation) the name of a person who has not actually engaged in the reported research is considered by many scientists to be unethical. One of the chief offenders in this practice is the institutional superior who insists that his or her name appear in the byline of every article produced in the department he or she supervises."

Avoid long lists of authors if at all reconcilable with keeping your job. The pressure on scientists to publish is no doubt the reason for long lists of authors. Only important or significant contributions to the investigation justifies authorship. It is not something used to reward people with, or a gesture of thanks. All contributors who do not qualify as authors must be thanked in the Acknowledgements.

\section{THE ABSTRACT}

The Abstract is compiled after the rest of the manuscript is completed, but is usually printed just after the list of authors. The Abstract must briefly and succinctly describe the main points of the article:

I. a short statement of the problem studied or the aim of the investigation,

2. the methods of investigation and statistical analysis,

3. the population or subjects studied, and

4. the main finding(s).

The usual length is $150-200$ words, but follow the instructions of the journal.

Many readers use the Abstract to determine whether they want to read the article, to keep it for later use, or to skip it. The Abstract must contain the information needed for this decision.

Abstracting journals and some computerized data bases take over the abstract as is for further dissemination to scientists. It is important to keep in mind that more people will read the Abstract separated from the rest of the article than together with it. It must be able to be read and interpreted on its own.

Please note that the Abstract is not the first paragraph of the Introduction. Abbreviations and newly created 


\section{EMS physiotherapy equipment from Medical Distributors(Pty) Ltd}

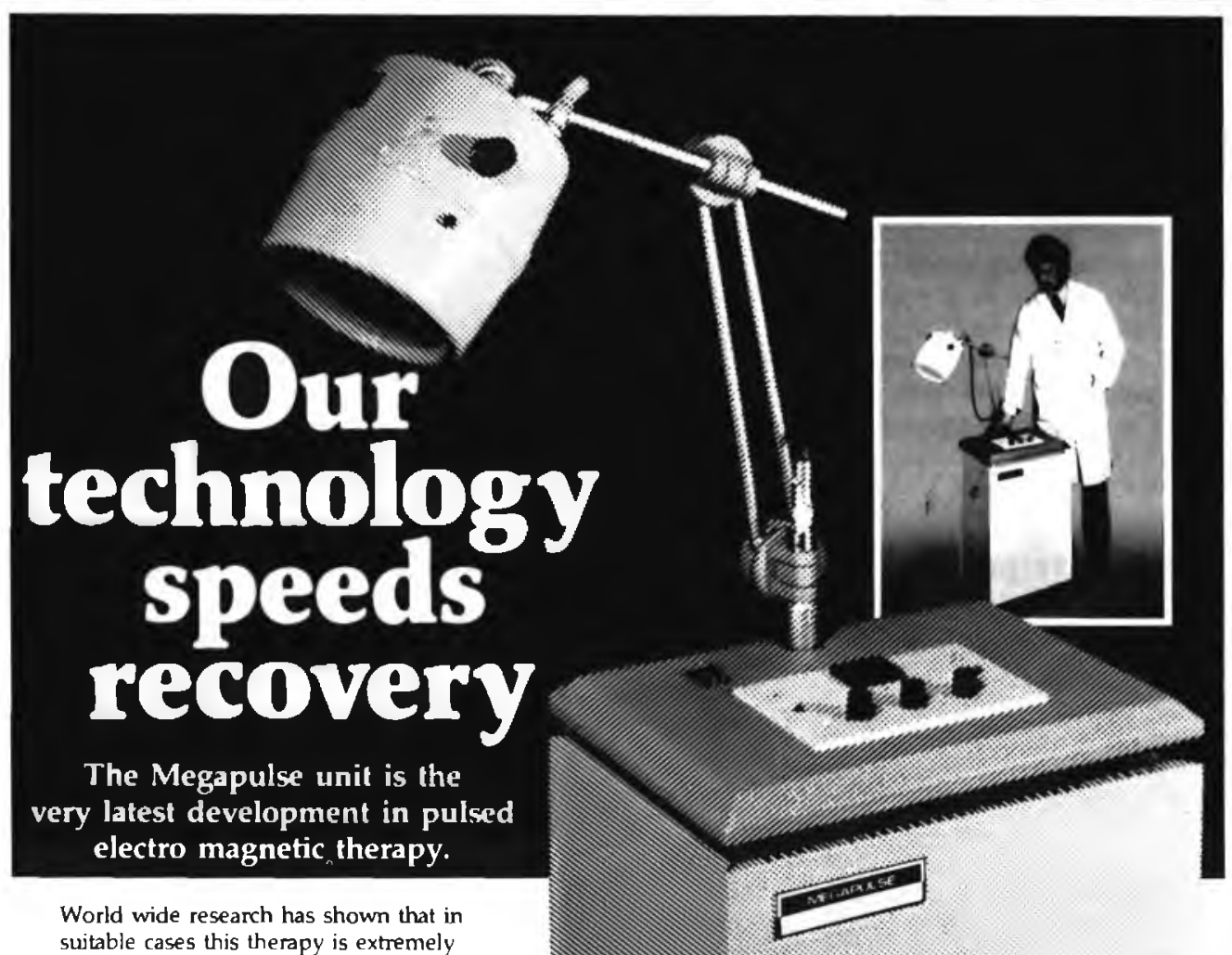

beneficial in speeding up natural healing processes.

With the Megapulse the therapist has avalable an increased range of pulse widths and repeat rates allowing a greater choice of treatments than ever before.

The Megapulse can be used to increase recovery rales when treating:-

- Inflammation - Tissue repairs

- Arterial Ulcers - Pressure sores

- Vascular, venous and orthopaedic conditions

Like all EMS equipment the Megapulse is reliable and superbly designed and is backed by an excellent after sales service

Detailed information on the Megapulse is

available from the address below:-

\section{MEDICAL DISTRIBUTORS ${ }_{E D M S B P K}^{P T Y}$}

P O Box 3378 Johannesburg 2000

P O Box 195 Cape Town 8000

P O Box 5298 Durban
Phone : 29-0611

Phone : $47-4440$

Phone : 37-1501 
terms used in the Abstract must be re-defined at the first mention in the text. The same remark goes for abbreviations and new terms in the title.

\section{THE INTRODUCTION}

The aims of a good introduction are:

1. to state the nature and extent of the problem investigated clearly,

2. to orientate the reader by reviewing the appropriate literature,

3. to identify gaps or contradictions in the present knowledge as it appears from the literature,

4. to review previous methods of investigation or study models and to justify or explain the one chosen by the author for the present investigation, and

5. to state some kind of a hypothesis, even a tentative one, on the basis of the study of the literature and what the author is looking for in the investigation. The days of a purely descriptive study undertaken with no statistical planning are in most cases long past.

These rules are well-known and need no elaboration. It is important to keep the literature review brief and to the point, because vague, wandering arguments here will leave the reader unimpressed with brilliant conclusions later. The introductory sentence is notoriously difficult to write and a bit of imagination can help to stimulate reader interest. Only resort to the standard "In 1979 Smith and Watson found that ..." if all else fails. Why fire your first shot under someone else's flag? A more useful way is to start with a sentence orientating the reader as to the broad topic the article is going to deal with: "In spite of the availability of effective drugs since 1970, the mortality due to ... disease is still unacceptably high in some rural areas of the country," or "Patients' views on sickness and understanding of treatment have an important influence on their compliance with a drug regimen."

Highly specialised concepts and original creations and systems of annotation can also be explained in the introduction.

The Introduction must form a complete unit - a whole - and must have an unbroken line of argument running through it. It must have a "story line" and should not just be a collection of jumbled facts and cited papers. Published work should only be cited to support or make a point in the line of argument. They must never be cited just because they deal roughly with the same subject or to make the reading list look impressive. The cited work must have direct bearing on the present study.

Be careful not to justify the experimental design or possible choices of experimental methods in the Discussion. Such justifications are usually more suited to the Introduction, since the reader should take note of them before starting on the Materials and Methods.

\section{MATERIALS AND METHODS}

Complete details of the experiment are given here. "Complete" is usually taken to mean as many details as a competent researcher in the field needs to repeat the experiment. To this I would like to add that details essential to the interpretation of the results must be mentioned even though the experiment may have been fully explained in a previous publication. Enough detail of previously published methods must be given so that the reader will have at least a superficial knowledge of the methods employed and the sample studied in order to read further without returning to the library. The amount of detail will depend on available space, how well-known the methods are, and the editorial policy of the journal. Rather give too many details than too few - the editor will take out superfluous statements and will never penalise the manuscript because of them.

It is essential to give a a brief outline or "plan" of the experiment early on in this section to orientate the reader and to provide an overview of the experimental design so that all the details can be fitted into the broader whole.

Many experienced researchers will not read this section unless the methods used deviate from generally accepted procedures. However, they will almost certainly scrutinise this section if something hinders them in the results. Authors cannot afford the loss of credibility that occurs when referees or readers find a mistake in the Materials and Methods section. Take critical care that this section is correct and complete. Should a referee feel that the experiments are unrepeatable from the information given or cannot be interpreted because of obvious gaps, no amount of breathtaking results will stop him from turning the paper down.

Subheadings can help the reader to group together details meaningfully. Try to use the same pattern of subheadings in the Discussion.

Avoid the common mistake of slipping in some of the results here.

\section{RESULTS}

This section is a really significant part of the manuscript. It is the proof around which the arguments are built.

Usually not all results are mentioned in words, only the significant or outstanding ones. The most compact form to give the bulk of the data is by means of tables. Do not present the same results in two different ways (i.e. tabular and graphic).

Remember in the choice of tables or graphs as opposed to words, that the first two are more expensive to typeset than text, but when selected with care, tables and graphs can represent data in compact form. Graphs are ideally suited to show trends or profiles and can contrast a large number of data points with each other. Graphs give a good overall picture at a glance, but accurate values can usually not be obtained from the published version. Three to four lines are the most that should be used in a graph; otherwise confusion results because of reduced size. Where actual values are needed to compare two sets of data, tables are better.

The title of a table must be self-explanatory and the table on its own must make sense. A table gives infor- 
mation so that the text can be shorter. It is not an embellishment and must serve a purpose to convey essential information. All tables must be referred to in the text.

Data can be represented horizontally or vertically in a table. However, "can" does not mean "should". Organise data (as a general rule) so that elements read "down" and not "across". The independent variables are usually given reading "down", i.e. in columns (as opposed to rows). Rotate a table by $90^{\circ}$ in any case of doubt and see if you do not gain in clarity and space.

Follow the journal instructions carefully in the preparation of tables and graphs, as well as other material such as sketches and photographs. When the manuscript is presented to a journal, this care taken can already give the manuscript a slight edge over other contenders for the available space.

\section{DISCUSSION}

This is one of the most difficult sections to write and the one section where many manuscripts stand or fall. Even though the data is valid and interesting, the manuscript will be rejected if the Discussion distorts or obscures the results. Remember that the onus of proof rests with the author.

It is a source of both mirth and sadness among editors that even the most strange or incorrect results can be explained away blithely by some ingenious authors. Please be careful (and conservative!), for your own reputation's sake, when faced with strange or deviant results.

The Discussion can usually be divided into three components which are interwoven to form a small essay:

1. Interpretation of the results given in the previous section. This forms the main part of the Discussion. How can the results be explained? Remember to be factual and not to drag in circumstantial evidence. Supporting hiterature can be quoted if needed. Refer to the previous section (Results) as well as the points emerging from the tables and graphs. Most important: relate how all this fits in with the hypothesis stated in the Introduction.

Some authors are tempted to discuss results which do not differ significantly from the control values or from each other because the data showed some "tendencies" towards differing (e.g. always slightly more positive). These authors sometimes provide detailed explanations for these supposed differences as if they were really observed. "Tendencies" can only serve as a guideline to redesigning the experiment for higher accuracy or for another approach to the problem in order to obtain the required degree of sensitivity to test these supposed differences statistically.

2. Advances in knowledge. How do the findings fit into the framework of existing knowledge? What are the implications of these findings for other disciplines or subject areas? How do the findings affect existing practices? Here claims of others (with references) can be discussed and differences of opinion can be reconciled.

3. Future solutions. Opinions concerning theory, future solutions, etc. can be discussed. During the course of the experiment many valuable lessons were learnt about certain methods, manoeuvres or experiments that did not work. Information on these problems and how they were overcome should also be shared with the research community. This can stimulate another researcher to do further research. Remember to keep this short.

\section{ACKNOWLEDGEMENTS}

This small section is actually very important. Technical and financial aid must always be acknowledged with thanks. To thank a senior who had little to do with the work in order to impress is unworthy, because flattery corrupts the giver as well as the receiver.

\section{REFERENCES}

Only cited references may appear in the reference list. Unpublished data, summaries, etc. should not be taken up in the reference list, but should be cited as personal comments in the text. This is usually done between brackets in the text, but some journals use footnotes for this purpose. Make sure when comments from personal letters, conversations, etc., are cited that the permission of the author/speaker is obtained beforehand. Some journals insist on receiving permission in writing from the originators of these personal comments before allowing such citations.

There are many styles of referencing in use. A sure way of getting the manuscript sent straight back is to ignore the instructions to authors. Take great care that the references are as correct as humanly possible. Should a referee discover some serious flaws here (especially those smacking of dishonesty or plagiarism) it places a question mark over the correctness of the whole manuscript. This is a chance not worth taking.

\section{SPECIAL NOTE ABOUT CASE REPORTS}

Note that the case report is a special form of the scientific article and many of the points needed for an experimental study fall away. The basic structure of a scientific article (Abstract, Introduction, Materials and Methods, Results, Discussion, References) always applies, although in contracted form and not necessarily with formal headings. Case reports are published in order to learn something, or for archival purposes to preserve information on rare or interesting cases for later referral. The case report will have a considerably better chance of appearing in print if, in the Discussion, some reason for knowing about this case, or some important point illustrated by it, can be given. Mere curiosity value is not enough for a journal with pressing space problems. 


\section{TYPING AND PRESENTATION OF THE MANUSCRIPT}

There are certain minimum physical requirements a manuscript must meet. A badly typed manuscript will in most cases not even be considered by the editor. Manuscripts must be typed, not written, on one side of the page and in keeping with the style of the journal. Use generous margins for notes by the editor and for marking up the manuscript for printing. Supply the required number of readable copies with all photographs, tables and so on attached.

\section{BEFORE THE ENVELOPE IS SEALED}

Ask a colleague to read through the whole manuscript as well as the addenda in one sitting as a final control. Catastrophes can still be rectified at this stage.

Remember to pack manuscripts firmly and to protect all photographs, artwork etc. with extra layers or cardboard. Do not underestimate the trauma caused by the postal services. Keep an extra copy and inquire when receipt is not acknowledged within a reasonable time.

Do not expect the manuscript to be accepted without a murmur. Suggested improvements or comments by the referees are not meant as a threat or to belittle. It is the research that counts, not the garment in which it was clothed. If the required changes are few, it pays to do them. If radical changes are suggested, sit back and think again. Some further work can be done to present a stronger case, the manuscript can be rewritten, or a different journal chosen. Remember that very few manuscripts presented to popular prestigious overseas journals are accepted without any changes.

Although one keeps on learning about this intricate job of writing up scientific results, it is consoling to remember that there is always an opportunity to do better next time!

\section{References}

1. DeBakey L. The scientific journal: Editorial policies and practices. St 1 Luis: C V Mosby, 1976.

2. Council for Biology Editors. Style Manual. A Guide for Authors, Editors, and Publishers in the Biological Sciences. 4th ed. Rockville Pike, Bethesda: Council for Biology Editors, 1978, p. 8.
For Orthopaedic Appliances, Surgical corsets,

Cervical collars,

Children's shoes \& boots,

Artificial limbs,

Latest in plastic modification.

For Hiring and selling of Hospital Equipment and

Sick room requisites -

Wheel chairs, walking aids,

Commodes,

Hospital beds,

Traction appiaratus

Visit the orthopaedic mechanicians

\section{A. C. MILLER \& Co.}

Technicians registered with the

S.A. Medical and Dental Council

275 BREE STRRET JOHANNESBURG

Telephone (011) 337-4763

P.O. Box 3412 Johannesburg

PHYSIOTHERAPISTS -

LOOK SMART!

\author{
METAL S.A.S.P. \\ BADGES are \\ now available \\ at R5,00 each. \\ If desired, branches \\ may order in bulk.
}

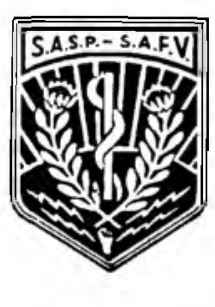

\section{Send cash with order to}

The Gen. Secretary, S.A.S.P.

P.O. Box 47238, Parklands

.2121 Johannesburg 